\title{
Neural Prescribed Performance Control for Uncertain Marine Surface Vessels without Accurate Initial Errors
}

\author{
Wenjie Si and Xunde Dong \\ School of Automation Science and Engineering, South China University of Technology, Guangdong 510640, China \\ Correspondence should be addressed to Xunde Dong; audxd@scut.edu.cn
}

Received 28 September 2016; Revised 14 November 2016; Accepted 22 November 2016; Published 15 January 2017

Academic Editor: Asier Ibeas

Copyright (C) 2017 W. Si and X. Dong. This is an open access article distributed under the Creative Commons Attribution License, which permits unrestricted use, distribution, and reproduction in any medium, provided the original work is properly cited.

\begin{abstract}
This paper deals with the problems concerned with the trajectory tracking control with prescribed performance for marine surface vessels without velocity measurements in uncertain dynamical environments, in the presence of parametric uncertainties, unknown disturbances, and unknown dead-zone. First, only the ship position and heading measurements are available and a high-gain observer is used to estimate the unmeasurable velocities. Second, by utilizing the prescribed performance control, the prescribed tracking control performance can be ensured, while the requirement for the initial error is removed via the preprocessing. At last, based on neural network approximation in combination with backstepping and Lyapunov synthesis, a robust adaptive neural control scheme is developed to handle the uncertainties and input dead-zone characteristics. Under the designed adaptive controller for marine surface vessels, all the signals in the closed-loop system are semiglobally uniformly ultimately bounded (SGUUB), and the prescribed transient and steady tracking control performance is guaranteed. Simulation studies are performed to demonstrate the effectiveness of the proposed method.
\end{abstract}

\section{Introduction}

As the demand for offshore exploration and operation, ocean surface vessels have been widely used in the marine industry. With the development of marine industrial technology, control design of marine surface vessels has become a hot topic of research $[1,2]$. Control of ocean surface ships is a difficult question; the working environment is often complex (ocean currents and sea breeze); external unpredictable disturbances may degrade control system performance and even damage the stability. Therefore, the tracking control of the marine vessels has attracted much attention.

Various tracking control approaches have been presented for marine surface vessels; the sliding mode tracking control schemes were developed in $[3,4]$ based on explicit models. For the systems with unknown system dynamics, both fuzzy logic systems (FLSs) and neural networks (NNs) have been proved to be useful in the control design, where their universal approximation properties are employed to model unknown nonlinear functions [5-12]. Recently, many approximation-based adaptive control schemes have also been proposed to handle the control problem for uncertain ocean surface ships [13-15]. The output feedback control and the full-state feedback control were designed based on approximation-based adaptive backstepping of the ship dynamics in [16]. A neural learning control method was presented in [17] to solve the problem of tracking control of unknown ship systems. In [18], the unknown ship dynamics was learned by using deterministic learning theory and then learning problem from neural output feedback control of uncertain ship dynamics was studied. The prescribed performance control was introduced in [19] to deal with the neural learning control of ocean surface vessels via deterministic learning. However, in the working environments of marine surface vessels, the initial errors cannot be obtained in advance, and the control method given by [19] is invalid in most working environments. To solve the aforementioned problem, we propose a new performance function for uncertain marine surface vessels with unknown initial errors.

Dead-zone is one of important input nonlinearities which appears in a wide range of practical engineering. The 
existence of dead-zone nonlinearities degrades the performance of control system and even may lead to system instability. In [20, 21], the robust adaptive control methods were used for nonlinear systems with parametric uncertainties subject to the input deal-zone, and the systems must satisfy linear parameterized condition. Recently, in order to deal with unknown nonlinear systems with input dead-zone when the knowledge of system functions is unavailable, many adaptive controllers have been proposed by utilizing universal approximation capability of neural networks or some fuzzy logic systems [21, 22]. A robust adaptive NN control design method was proposed in [23] for a kind of strict-feedback nonlinear systems with uncertainties and input dead-zone. An adaptive fuzzy output feedback control was studied in [24] for switched nonlinear systems with uncertainties. In [25], the problem of the adaptive fuzzy backstepping output feedback tracking control was investigated for multi-input and multioutput (MIMO) stochastic nonlinear systems. The problem of adaptive decentralized NN control was investigated in [26] for large-scale stochastic nonlinear time-delay systems with input dead-zone.

In the control design, the tracking error is only required to converge to a small residual set, while the transient and steady-state tracking performance is not considered. The practical engineering often requires certain prespecified performance. More recently, the prescribed performance control (PPC) has been proposed in [27]. Furthermore, in [28, 29], the PPC was used for the position tracking control of robot. Combining PPC with dynamic surface control (DSC), a fuzzy control scheme was studied in [30] to ensure the performance of tracking control. When states were unmeasurable, an output feedback control was presented in [31] for large-scale nonlinear time-delay systems. The prescribed performance control technology was extended to MIMO systems [32, 33]. However, to our best knowledge, by using prescribed performance control, no tracking control methods exit for uncertain ocean surface vessels without the need for the initial error conditions.

Motivated by the aforementioned discussion, to guarantee the predefined performance for ocean surface vessels without velocity measurements in the presence of unknown input dead-zone, we will design an adaptive neural output feedback control scheme. RBF NNs are used to approximate the unknown nonlinearities. The prescribed performance function is designed to ensure the performance of the prescribed tracking control without any consideration for accurate initial errors. Then, based on the backstepping and Lyapunov theory, we propose an adaptive neural tracking control method to ensure the boundedness of the closed-loop system.

Compared with previous works, our paper has the following advantages. (1) The unmeasurable velocities of the ocean surface ship are estimated by employing a high-gain observer. To prevent peaking of the high-gain observer at the initial phase, a new method is used different from the saturation functions employed in [16, 34]. (2) A performance function is given to ensure the tracking control performance, and the requirement for the exact initial error is removed. (3) With the proposed controller, the tracking control for uncertain surface vessels is achieved with only position sensors and the control performance of the system is guaranteed by prescribed performance control.

The rest of the paper is organized as follows. Section 2 presents the preliminaries and problem formulation. An adaptive neural tracking control scheme for uncertain ocean surface vessels with prescribed performance control is given in Section 3. The simulation studies are presented in Section 4 to demonstrate the effectiveness of the proposed method. Section 5 concludes this paper.

\section{Problem Formulation and Preliminaries}

2.1. Ship Dynamics. Consider multiple-input-multipleoutput (MIMO) systems for a three degrees of freedom surface vessel subject to unknown model uncertainties and input dead-zone. The dynamics of the surface vessel is described by $[1,35]$

$$
\begin{aligned}
& \dot{\eta}=J(\eta) \nu \\
& \tau=M \dot{v}+C(\nu) \nu+D(\nu) \nu+g(\eta)+\Delta(\eta, v),
\end{aligned}
$$

where $\eta=[x, y, \psi]^{T} \in R^{3},(x, y)$ is the surface vessel position, and $\psi$ is the surface vessel heading; $J(\eta)$ is the rotation matrix; $v=[u, v, r]^{T} \in R^{3}, u, v, r$ are velocities of the surge, the sway, and the yaw, respectively; $\tau \in$ $R^{3}$ is the vector of control input, $M$ denotes the inertia matrix of the ship, $C(\nu)$ is the total Coriolis and centripetal acceleration matrix, $D(\nu)$ is the damping matrix, $g(\eta)=$ $\left[g_{1}(\eta), g_{2}(\eta), g_{3}(\eta)\right]^{T}$ is the vector of buoyancy/gravitational forces, and $\Delta(\eta, v)=\left[\Delta_{1}(\eta, v), \Delta_{2}(\eta, v), \Delta_{3}(\eta, \nu]^{T}\right.$ is used to model the uncertainties with $\Delta_{i}(\eta, \nu),(i=1,2,3)$ being unknown.

Precisely, $M=M^{T}>0$ is given by [35]; $J(\eta), C(\nu)$, and $D(\nu)$ are given as follows:

$$
\begin{aligned}
& J(\eta)=\left[\begin{array}{ccc}
\cos \psi & -\sin \psi & 0 \\
\sin \psi & \cos \psi & 0 \\
0 & 0 & 1
\end{array}\right], \\
& C(\nu)=\left[\begin{array}{ccc}
0 & 0 & c_{13}(\nu) \\
0 & 0 & c_{23}(\nu) \\
-c_{13}(\nu) & -c_{23}(\nu) & 1
\end{array}\right], \\
& D(\nu)=\left[\begin{array}{ccc}
d_{11}(\nu) & 0 & 0 \\
0 & d_{22}(\nu) & d_{23}(\nu) \\
0 & d_{32}(\nu) & d_{33}(\nu)
\end{array}\right],
\end{aligned}
$$

where $c_{13}(\nu), c_{23}(\nu), d_{11}(\nu), d_{22}(\nu), d_{23}(\nu), d_{32}(\nu)$, and $d_{33}(\nu)$ are unknown. 
We have the description of the ship dynamics (1) subject to the unknown dead-zone nonlinearity as follows:

$$
\begin{aligned}
& \dot{\eta}=J(\eta) v \\
& \dot{v}=M^{-1}(-C(\nu) \nu-D(\nu) v-g(\eta)+\tau-\Delta(\eta, v)) \\
& \tau=\mathscr{D}\left(u_{\tau}\right),
\end{aligned}
$$

where $\eta \in \mathbf{R}^{3}$ is the system output, $\tau \in \mathbf{R}^{3}$ is the system input and the dead-zone output, and the actuator dead-zone characteristic is described as $\mathscr{D}\left(u_{\tau}\right)$ with $u_{\tau}$ being the input of the unknown dead-zone.

The main goal of this control scheme is to present an adaptive NN tracking controller for the system described by (3) to ensure that the system output $\eta \in \mathbf{R}^{3}$ can track the reference signal $y_{d}(t) \in \mathbf{R}^{3}$ and all signals in the closed-loop system remain bounded.

Assumption 1. The system output $\eta(t) \in \mathbf{R}^{3}$ and its first-order derivative $\dot{\eta}(t) \in \mathbf{R}^{3}$ are continuous and bounded, such that $\|\eta\|<Y_{0}$ and $\|\dot{\eta}\|<Y_{1}$, where $Y_{0}$ and $Y_{1}$ are positive constants.

Assumption 2. The reference signal $y_{d}(t) \in \mathbf{R}^{3}$ and its $n$th order $y_{d}^{n}(t) \in \mathbf{R}^{3}$ are continuous and bounded.

2.2. Dead-Zone Characteristic. The actuator dead-zone nonlinearity can be described as follows [36]:

$$
\tau=\mathscr{D}\left(u_{\tau}\right)= \begin{cases}h_{r}\left(u_{\tau}-b_{r}\right) & u_{\tau} \geq b_{r} \\ 0 & b_{l}<u_{\tau}<b_{r} \\ h_{l}\left(u_{\tau}-b_{l}\right) & u_{\tau} \leq b_{l},\end{cases}
$$

where $h_{r}(\cdot)$ and $h_{l}(\cdot)$ are unknown smooth functions.

Assumption 3. The parameters of the dead-zone $b_{r}$ and $b_{l}$ are unknown constants and satisfy $b_{r}>0$ and $b_{l}<0$.

Assumption 4. For unknown functions $h_{r}(\cdot)$ and $h_{l}(\cdot)$, there exist unknown constants $H_{0}$ and $H_{1}$, such that

$$
\begin{aligned}
& 0<H_{r 0}<\dot{h}_{r}\left(u_{\tau}-b_{r}\right)<H_{r 1}, \quad r \in\left[b_{r},+\infty\right] \\
& 0<H_{l 0}<\dot{h}_{l}\left(u_{\tau}-b_{l}\right)<H_{l 1}, \quad r \in\left(-\infty, b_{l}\right] .
\end{aligned}
$$

Let $\mathscr{D}_{\min }=\min \left\{H_{0}\right\}$ and $\mathscr{D}_{\max }=\max \left\{H_{1}\right\}$. The deadzone nonlinearity can be rewritten as

$$
\tau=\mathscr{D}\left(u_{\tau}\right)=\Xi\left(u_{\tau}\right) u_{\tau}+d\left(u_{\tau}\right),
$$

where

$$
\begin{gathered}
\Xi= \begin{cases}\Xi_{r}^{\prime} & u_{\tau} \geq b_{r} \\
\Xi_{l}^{\prime} & u_{\tau}<b_{r},\end{cases} \\
d\left(u_{\tau}\right)= \begin{cases}-\Xi_{r}^{\prime} b_{r} & u_{\tau} \geq b_{r} \\
-\Xi_{r}^{\prime} u_{\tau} & b_{r}<u_{\tau}<b_{l} \\
-\Xi_{l}^{\prime} b_{l} & u_{\tau} \leq b_{l},\end{cases}
\end{gathered}
$$

where $\Xi_{r}^{\prime}=\dot{h}_{r}(\cdot)$ and $\Xi_{l}^{\prime}=\dot{h}_{l}(\cdot)$.

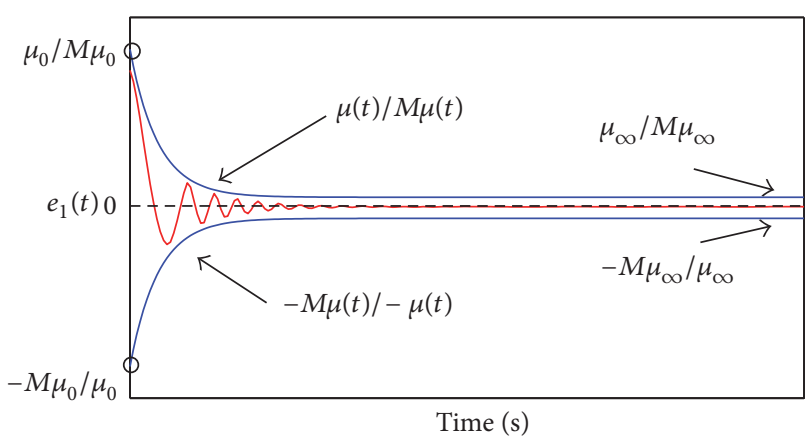

FIGURE 1: Prescribed performance of the error $e_{1}(t)$.

It can be obtained that $\mathscr{D}_{\text {min }} \leq \Xi \leq \mathscr{D}_{\max },\|d(u)\| \leq d^{*}:=$ $\max \left\{-\mathscr{D}_{\max } d_{1}, \mathscr{D}_{\max } d_{r}\right\}$.

2.3. Prescribed Performance. This section introduces the concept of the prescribed performance. Then, a performance function is given and it will be chosen to be used in the control design.

Definition 5 (see [27]). A smooth function $\rho(t): R^{+} \rightarrow R^{+}$is called a performance function, if

(i) $\rho(t)$ is a strictly positive decreasing function;

(ii) $\lim _{t \rightarrow+\infty} \rho(t)=\rho_{\infty}>0$, where $\rho_{\infty}$ is a positive constant.

According to Definition 5, we choose the performance function as follows:

$$
\mu(t)=\left(\mu_{0}-\mu_{\infty}\right) \exp (-\kappa t)+\mu_{\infty},
$$

where $\mu_{0}, \mu_{\infty}$ and $\kappa$ are design parameters. It is obvious that $\mu(t)$ satisfies that $\lim _{t \rightarrow \infty} \mu(t)=\mu_{\infty}$. According to the description of the performance function $\mu(t)$, the tracking error $e_{1}=y-y_{d}$ has the following performance bound (as shown in Figure 1):

$$
\begin{aligned}
-M \mu(t) & <e_{1}(t)<\mu(t), \quad \text { if } e_{1}(t) \geq 0 \\
-\mu(t) & <e_{1}(t)<M \mu(t), \quad \text { if } e_{1}(t)<0,
\end{aligned}
$$

where $0 \leq M \leq 1, t \geq 0$.

To represent (9) by an unconstrained form, the following state transformation is employed [27]:

$$
e_{1}(t)=\mu(t) R\left(\zeta_{1}\right)
$$

where

$$
\begin{aligned}
& R\left(\zeta_{1}\right)=M \frac{e^{\zeta_{1}}-e^{-\zeta_{1}}}{M e^{\zeta_{1}}+e^{-\zeta_{1}}}, \quad \text { if } e_{1}(t) \geq 0 \\
& R\left(\zeta_{1}\right)=M \frac{e^{\zeta_{1}}-e^{-\zeta_{1}}}{e^{\zeta_{1}}+M e^{-\zeta_{1}}}, \quad \text { if } e_{1}(t)<0
\end{aligned}
$$


and $\zeta_{1}$ is called the transformed error. According to (11), the derivation of $\zeta_{1}$ is as follows:

$$
\dot{\zeta}_{1}=\frac{\partial R^{-1}}{\partial \omega} \dot{\omega}= \begin{cases}\frac{1}{2} \frac{1+M}{(\omega+M)(1-\omega)} \dot{\omega}, & \text { if } e_{1}(t) \geq 0 \\ \frac{1}{2} \frac{1+M}{(\omega+1)(M-\omega)} \dot{\omega}, & \text { if } e_{1}(t)<0 .\end{cases}
$$

Based on $e_{1}(t)=x_{1}(t)-y_{d}$, one has

$$
\begin{aligned}
\dot{\omega} & =\frac{1}{\mu(t)}\left(\dot{e}_{1}-\frac{\dot{\mu}(t)}{\mu(t)} e_{1}(t)\right), \\
\dot{\zeta}_{1} & =\frac{1}{\mu} \frac{\partial R^{-1}}{\partial \omega}\left(\dot{e}_{1}-\frac{\dot{\mu}(t)}{\mu(t)} e_{1}(t)\right) \\
& =p_{1}\left(\dot{e}_{1}-\frac{\dot{\mu}(t)}{\mu(t)} e_{1}(t)\right)
\end{aligned}
$$

with

$$
p_{1}=\frac{1}{\mu} \frac{\partial R^{-1}}{\partial \omega}
$$

2.4. RBF Neural Networks. It has been shown that neural networks are good at modeling unknown nonlinear functions in control design [37]. In the study, RBF NN will be employed to model unknown continuous function $f(Z): R^{q} \rightarrow R$ over a compact set $\Omega_{Z} \subset R^{q}$ for a given arbitrary accuracy $\epsilon^{*}>0$ as follows:

$$
f(Z)=W^{* T} S(Z)+\epsilon(Z),
$$

where $Z \in \Omega_{Z}$ is the input vector with $q$ being the input dimension of the neural networks. $W=\left[w_{1}, w_{2}, \ldots, w_{l}\right]^{T} \in$ $R^{l}$ is the neural weight vector with $l>1$ being the node number of the neural network, and $W^{*}$ denotes the ideal constant weight vector. $\epsilon(Z)$ is the approximation error, $\|\epsilon(Z)\| \leq \epsilon^{*} . S(Z)=\left[s_{1}(Z), s_{2}(Z), \ldots, s_{l}(Z)\right]^{T}$ is the vector of basis function; $s_{i}(Z)$ commonly is Gaussian function which has the following form:

$$
s_{i}(Z)=\exp \left[\frac{-\left(Z-\xi_{i}\right)^{T}\left(Z-\xi_{i}\right)}{\eta^{2}}\right], \quad i=1, \ldots, l,
$$

where $\xi_{i}=\left[\xi_{i 1}, \xi_{i 2}, \ldots, \xi_{i q}\right]^{T}$ is the center of the receptive field and $\eta$ is the bandwidth of Gaussian function [37].

The ideal constant weight vector is defined as

$$
W^{*}:=\arg \min _{\widehat{W} \in R^{R}}\left\{\sup _{Z \in \Omega_{Z}}\left|f(Z)-\widehat{W}^{T} S(Z)\right|\right\},
$$

where $\widehat{W}$ is the estimation of $W^{*}$.
Lemma 6 (see [38]). Consider the Gaussian RBF neural networks (15). Let $\varrho:=(1 / 2) \min _{i \neq j}\left\|\xi_{i}-\xi_{j}\right\| . q$ is the dimension of the neural network input $Z$ and $\eta$ is the width of the Gaussian function (16); then the following inequality holds:

$$
\|S(Z)\| \leq \sum_{k=0}^{\infty} 3 q(k+2)^{q-1} e^{-2 e^{2} k^{2} / \eta^{2}}:=s^{*},
$$

where $s^{*}$ is an upper bound of $\|S(Z)\|$ and is limited and independent of neural input $Z$ and the neural weights dimension $l$.

2.5. High-Gain Observer. The system output $\eta=[x, y, \psi]^{T} \in$ $R^{3}$ is available for measurement, and $v=[u, v, r]^{T} \in R^{3}$ is unavailable. To solve the unmeasured states problem, we need a high-gain observer used in [39].

Lemma 7 (see [16]). Consider the following linear system:

$$
\begin{aligned}
& \varepsilon_{\lambda} \dot{\pi}_{1}=\pi_{2}, \\
& \varepsilon_{\lambda} \dot{\pi}_{2}=-\lambda \pi_{2}-\pi_{1}+\eta,
\end{aligned}
$$

where $\varepsilon_{\lambda}$ is a small constant, $\pi_{1}$ and $\pi_{2}$ are system states, and $\lambda$ is chosen to ensure that the linear system is stable. According to Assumption (1), the following properties hold:

(i)

$$
\frac{\pi_{2}}{\varepsilon_{\lambda}}-\dot{\eta}=-\varepsilon_{\lambda} \ddot{\phi}
$$

where $\phi=\pi_{2}+\lambda \pi_{1}$.

(ii) There exist two constants $t^{*}>0$ and $h_{0}>0$, which rely on the parameters $Y_{0}, Y_{1}, \varepsilon_{\lambda}$, and $\lambda$, such that $\|\ddot{\phi}\| \leq h_{0}$, $\forall t>t^{*}$.

Considering that $\dot{\eta}=J(\eta) v$ in system (1) and the property $J^{T}(\eta) J(\eta)=I$, we can use $\widehat{v}=J^{T}(\eta)\left(\pi_{2} / \varepsilon_{\lambda}\right)$ to estimate the state variable $\nu$.

Lemma 8 (see [39]). For the RBF neural networks, if $Z=\widehat{Z}-$ $\varepsilon \phi, \varepsilon>0, \phi$ is a bounded vector; then

$$
S(\widehat{Z})=S(Z)+\varepsilon S_{t},
$$

where $S_{t}$ is the bounded function, $\left\|S_{t}\right\|<s_{t}^{*}$; $s_{t}^{*}$ is a positive constant.

\section{Adaptive Neural Control Design}

The backstepping design is based on the following coordinate changes:

$$
\begin{aligned}
& z_{1}=\zeta_{1}, \\
& z_{2}=v-\alpha_{1},
\end{aligned}
$$

where $\alpha_{1}$ is the virtual control signal, which is defined later. 
Step 1. Considering $e_{1}(t)=\eta(t)-y_{d}$ and $\dot{e}_{1}=J(\eta) \nu-\dot{y}_{d}$, one has

$$
\dot{z}_{1}(t)=p_{1}\left(J(\eta) \nu-\dot{y}_{d}-\frac{\dot{\mu}}{\mu} e_{1}\right) .
$$

Noting the following the error variable as $z_{2}=\nu-\alpha_{1}$, we have

$$
\dot{z}_{1}(t)=p_{1}\left(J(\eta)\left(z_{2}+\alpha_{1}\right)-\dot{y}_{d}-\frac{\dot{\mu}}{\mu} e_{1}\right) .
$$

From the property $J^{T}(\eta) J(\eta)=I, \alpha_{1}$ is defined as

$$
\alpha_{1}=J^{T}(\eta)\left(-K_{1} p_{1}^{-1} z_{1}+\dot{y}_{d}+\frac{\dot{\mu}}{\mu} e_{1}\right),
$$

where $K_{1}^{T}=K_{1} \geq 0$ is a design parameter.

We choose a Lyapunov function candidate $V_{1}=$ $(1 / 2) z_{1}^{T} z_{1}$, and its derivative along (24) yields

$$
\begin{aligned}
\dot{V}_{1} & =z_{1}^{T} p_{1}\left(J(\eta) z_{2}-K_{1} p_{1}^{-1} z_{1}\right) \\
& =-z_{1}^{T} K_{1} z_{1}+z_{1}^{T} p_{1} J(\eta) z_{2},
\end{aligned}
$$

where the last term $z_{1}^{T} p_{1} J(\eta) z_{2}$ will be canceled in the next step.

Step 2. From $z_{2}=v-\alpha$, we have

$$
\begin{aligned}
\dot{z}_{2}= & M^{-1}(-C(\nu) \nu-D(\nu) \nu-g(\eta)+\tau-\Delta(\eta, \nu)) \\
& -\dot{\alpha} .
\end{aligned}
$$

Noting $M^{T}=M \geq 0$ is the design constant obtained by [35], we consider the following Lyapunov function candidate:

$$
V_{2}=\frac{1}{2} z_{2}^{T} M z_{2}
$$

The derivative of $V_{2}$ along (27) is obtained by

$$
\begin{gathered}
\dot{V}_{2}=z_{2}^{T}(-C(\nu) v-D(\nu) v-g(\eta)+\tau-\Delta(\eta, v) \\
-M \dot{\alpha})=z_{2}^{T}\left(\Xi\left(u_{\tau}\right) u_{\tau}+d\left(u_{\tau}\right)-C(\nu) \nu\right. \\
-D(\nu) \nu-g(\eta)-\Delta(\eta, \nu)-M \dot{\alpha}) .
\end{gathered}
$$

Denote

$F(Z)$

$$
=-(C(\nu) \nu+D(\nu) \nu+g(\eta)+\Delta(\eta, \nu)+M \dot{\alpha}),
$$

where $F(Z)=\left[f_{1}(Z), f_{2}(Z), f_{3}(Z)\right]^{T} \in R^{3}, Z=[\eta, \nu, \alpha, \dot{\alpha}] \epsilon$ $R^{8}$. A RBF NN $W^{* T} S$ is employed to approximate the unknown function $F(Z)$ as follows:

$$
F(Z)=W^{* T} S(Z)+\epsilon(Z), \quad|\epsilon(Z)| \leq \epsilon^{*},
$$

where $W^{* T} S\left(Z_{1}\right)=\left[W_{1}^{* T} S_{1}(Z), W_{2}^{* T} S_{2}(Z), W_{3}^{* T} S_{3}(Z)\right]^{T} \epsilon$ $R^{3}$ and $\epsilon(Z)=\left[\epsilon_{1}(Z), \epsilon_{2}(Z), \epsilon_{3}(Z)\right]^{T} \in R^{3}$ is the approximation error.

We choose the following control as

$$
u_{\tau}=\frac{1}{C_{1}}\left(-K_{2} \widehat{z}_{2}-\widehat{W}^{T} S(\widehat{Z})-J(\eta) p_{1} z_{1}\right),
$$

where $\widehat{Z}=[\eta, \widehat{v}, \alpha, \dot{\alpha}] \in R^{8} . \widehat{W}^{T} S(\widehat{Z})=\left[\widehat{W}_{1}^{T} S_{1}(\widehat{Z}), \widehat{W}_{2}^{T} S_{2}(\widehat{Z})\right.$, $\left.\widehat{W}_{3}^{T} S_{3}(\widehat{Z})\right]^{T} \in R^{3} ; C_{1}=D_{\max }$ is a design parameter.

From Lemma 7 and the property $J^{T}(\eta) J(\eta)=I$, one has

$$
\begin{aligned}
\widetilde{z}_{2} & =\widehat{z}_{2}-z_{2}=J^{T}(\eta) \frac{\pi_{2}}{\varepsilon_{\lambda}}-\alpha-(\nu-\alpha) \\
& =J^{T}(\eta) \frac{\pi_{2}}{\varepsilon_{\lambda}}-\nu=J^{T}(\eta)\left(\frac{\pi_{2}}{\varepsilon_{\lambda}}-\dot{\eta}\right)=-J^{T}(\eta) \varepsilon_{\lambda} \ddot{\phi} \\
& =\beta,
\end{aligned}
$$

where $\|\beta\| \leq \bar{\varepsilon}_{\lambda} h_{0} \cdot \bar{\varepsilon}_{\lambda}=\left\|J^{T}(\eta) \varepsilon_{\lambda}\right\|>0$ is constant.

According to Lemmas 7 and 8 , we have

$$
\begin{aligned}
S_{i}(\widehat{Z}) & =S_{i}(Z)+\varepsilon S_{i t} \\
\widehat{W}_{i}^{T} S_{i}(\widehat{Z}) & =W_{i}^{* T} S_{i}(Z)+W_{i}^{* T} \varepsilon S_{i t}+\widetilde{W}_{i}^{T} S_{i}(\widehat{Z}) .
\end{aligned}
$$

Consider the following Lyapunov function candidate

$$
\begin{aligned}
V & =V_{1}+V_{2}+\frac{1}{2} \sum_{i=1}^{3} \widetilde{W}_{i}^{T} \Gamma_{i}^{-1} \widetilde{W}_{i} \\
& =\frac{1}{2} z_{1}^{T} z_{1}+\frac{1}{2} z_{2}^{T} M z_{2}+\frac{1}{2} \sum_{i=1}^{3} \widetilde{W}_{i}^{T} \Gamma_{i}^{-1} \widetilde{W}_{i} .
\end{aligned}
$$

The time derivative of $V$ is given by

$$
\begin{aligned}
\dot{V} & =-z_{1}^{T} K_{1} z_{1}+z_{1}^{T} p_{1} J(\eta) z_{2}+z_{2}^{T}\left(\Xi\left(u_{\tau}\right) u_{\tau}+d\left(u_{\tau}\right)\right. \\
& -C(\nu) \nu-D(\nu) \nu-g(\eta)-\Delta(\eta, v)-M \dot{\alpha}) \\
& +\sum_{i=1}^{3} \widetilde{W}_{i}^{T} \Gamma_{i}^{-1} \dot{\widetilde{W}}_{i} .
\end{aligned}
$$


Define the adaptive law as follows:

$$
\dot{\widehat{W}}=\dot{\bar{W}}=\Gamma\left[S(\widehat{Z}) \widehat{z}_{2}-\sigma \widehat{W}\right]
$$

where $\Gamma=\Gamma^{T}>0$ and $\sigma$ are design parameters.

Substituting (32) and (37) into (36) yields

$$
\begin{aligned}
\dot{V} \leq & -z_{1}^{T} K_{1} z_{1}+z_{1}^{T} p_{1} J(\eta) z_{2}+z_{2}^{T}\left(-K_{2} \widehat{z}_{2}\right. \\
& \left.-\widehat{W}^{T} S(\widehat{Z})-p_{1} J(\eta) z_{1}+W^{* T} S(Z)+\epsilon\right)+z_{2}^{T} d^{*} \\
& +\widetilde{W}^{T}\left[S(\widehat{Z}) \widehat{z}_{2}-\sigma \widehat{W}\right] \leq-z_{1}^{T} K_{1} z_{1}-z_{2}^{T} K_{2} z_{2} \\
& -z_{2}^{T} K_{2} \widetilde{z}_{2}-\sum_{i=1}^{3} z_{2, i} W_{i}^{* T} \varepsilon S_{i t}-\sum_{i=1}^{3} z_{2, i} \widetilde{W}_{i}^{T} S_{i}(\widehat{Z}) \\
& +z_{2}^{T} \epsilon+z_{2}^{T} d^{*}+\widetilde{W}^{T}\left[S(\widehat{Z}) \widehat{z}_{2}-\sigma \widehat{W}^{T}\right] \leq-z_{1}^{T} K_{1} z_{1} \\
& -z_{2}^{T} K_{2} z_{2}-z_{2}^{T} K_{2} \widetilde{z}_{2}-\sum_{i=1}^{3} z_{2, i} W_{i}^{* T} \varepsilon S_{i t} \\
& +\sum_{i=1}^{3} \widetilde{z}_{2, i} \widetilde{W}_{i}^{T} S_{i}(\widehat{Z})+z_{2}^{T} \epsilon+z_{2}^{T} d^{*}-\sigma \widetilde{W}^{T} \widehat{W} .
\end{aligned}
$$

Considering $\widetilde{W}=\widehat{W}-W^{*}$, we have

$$
\begin{aligned}
-\sigma \widetilde{W}^{T} \widehat{W} & =-\sigma \widetilde{W}^{T}\left(W^{*}+\widetilde{W}\right) \\
& \leq-\sigma\|\widetilde{W}\|^{2}+\sigma\|\widetilde{W}\|\left\|W^{*}\right\| \\
& \leq \frac{1}{2} \sigma\left(\left\|W^{*}\right\|^{2}-\|\widetilde{W}\|^{2}\right) .
\end{aligned}
$$

By completing the squares, we have

$$
\begin{gathered}
z_{2}^{T} \epsilon \leq \frac{1}{2} z_{2}^{T} z_{2}+\frac{1}{2}\left\|\epsilon^{*}\right\|^{2} \\
z_{2}^{T} d^{*} \leq \frac{1}{2} z_{2}^{T} z_{2}+\frac{1}{2} d^{* 2} \\
-\sum_{i=1}^{3} z_{2, i} W_{i}^{* T} \varepsilon S_{i t} \leq \frac{1}{2} z_{2}^{T} z_{2}+\sum_{i=1}^{3} \frac{W_{i}^{*} \varepsilon^{2}\left\|S_{i t}\right\|^{2}}{2} \\
\sum_{i=1}^{3} \widetilde{z}_{2, i} \widetilde{W}_{i}^{T} S_{i}(\widehat{Z}) \leq \sum_{i=1}^{3} \frac{\sigma_{i}\left\|\widetilde{W}_{i}\right\|^{2}}{4}
\end{gathered}
$$$$
+\sum_{i=1}^{3} \frac{2\left\|S_{i}(\widehat{Z})\right\|^{2}}{\sigma_{i}} \frac{1}{2} \widetilde{z}_{2}^{T} \widetilde{z}_{2}
$$$$
-z_{2}^{T} K_{2} \widetilde{z}_{2} \leq \frac{1}{2} z_{2}^{T} z_{2}+\frac{1}{2}\left(K_{2} \tilde{z}_{2}\right)^{T}\left(K_{2} \widetilde{z}_{2}\right) .
$$

Substituting (39)-(40) into (38) yields

$$
\begin{aligned}
\dot{V} \leq & -z_{1}^{T} K_{1} z_{1}-z_{2}^{T}\left(K_{2}-2 I\right) z_{2}-\frac{1}{4} \sigma\|\widetilde{W}\|^{2} \\
& +\frac{1}{2}\left\|\epsilon^{*}\right\|^{2}+\frac{1}{2} d^{* 2}+\frac{1}{2} \sum_{i=1}^{3}\left(\varepsilon_{i}^{2}\left\|S_{i t}\right\|^{2}+\sigma_{i}\right)\left\|W_{i}^{*}\right\|^{2} \\
& +\lambda_{\max }\left(K_{2}^{T} K_{2}+\operatorname{diag}\left[\frac{2 s^{* 2}}{\sigma_{i}}\right]\right) \frac{1}{2} \beta^{T} \beta,
\end{aligned}
$$

where $\lambda_{\max }(A)$ and $\lambda_{\text {min }}(A)$ denote the minimum and maximum eigenvalues of matrix $A$.

Considering $(1 / 2) \beta^{T} \beta \leq(1 / 2) \bar{\varepsilon}_{\lambda}^{2} h_{0}^{2}$, we have

$$
\begin{aligned}
\dot{V} \leq & -z_{1}^{T} K_{1} z_{1}-z_{2}^{T}\left(K_{2}-2 I\right) z_{2}-\frac{1}{4} \sigma\|\widetilde{W}\|^{2} \\
& +\frac{1}{2}\left\|\epsilon^{*}\right\|^{2}+\frac{1}{2} d^{* 2}+\frac{1}{2} \sum_{i=1}^{3}\left(\varepsilon_{i}^{2}\left\|S_{i t}\right\|^{2}+\sigma_{i}\right)\left\|W_{i}^{*}\right\|^{2} \\
& +\lambda_{\max }\left(K_{2}^{T} K_{2}+\operatorname{diag}\left[\frac{2 s^{* 2}}{\sigma_{i}}\right]\right) \frac{1}{2} \bar{\varepsilon}_{\lambda}^{2} h_{0}^{2},
\end{aligned}
$$

where diag $[*]$ denotes a block-diagonal matrix.

Then, we have the following inequality:

$$
\dot{V} \leq-\rho_{1} V+\delta_{1}
$$

where

$$
\begin{aligned}
\rho_{1} & =\min \left\{2 \lambda_{\min }\left(K_{1}\right), \frac{2 \lambda_{\min }\left(K_{2}-2 I\right)}{\lambda_{\max }(M)},\right. \\
\left.\min _{i=1,2, \ldots, n} \frac{\sigma_{i}}{2 \lambda_{\max }\left(\Gamma_{i}^{-1}\right)}\right\} & \\
\delta_{1} & =\frac{1}{2}\left\|\epsilon^{*}\right\|^{2}+\frac{1}{2} d^{* 2}+\frac{1}{2} \sum_{i=1}^{3}\left(\varepsilon_{i}^{2}\left\|S_{i t}\right\|^{2}+\sigma_{i}\right)\left\|W_{i}^{*}\right\|^{2} \\
& +\lambda_{\max }\left(K_{2}^{T} K_{2}+\operatorname{diag}\left[\frac{2 s^{* 2}}{\sigma_{i}}\right]\right) \frac{1}{2} \bar{\varepsilon}_{\lambda}^{2} h_{0}^{2} .
\end{aligned}
$$

To ensure $\rho_{1}>0$, the parameters $K_{1}$ and $K_{2}$ are chosen to satisfy that $K_{1}>0$ and $K_{2}-2 I>0$.

Theorem 9. Consider the ship system composed of system (1), observer (19), the neural controller (32), and the adaptive law (37). Under Assumptions 1 and 2, if the initial conditions are bounded, then all the signals in the closed-loop system are semiglobally uniformly ultimately bounded. Moreover, for all $t \geq 0$ the tracking error $e_{1}=\eta-y_{d}$ is confined within the prescribed performance bounds. 
Proof. Multiplying (43) by $e^{\rho_{1} t}$ yields

$$
\frac{d}{d t}\left(V e^{\rho_{1} t}\right) \leq \delta_{1} e^{\rho_{1} t}
$$

Integrating (45), one has

$$
V \leq\left(V(0)-\frac{\delta_{1}}{\rho_{1}}\right) e^{-\rho_{1} t}+\frac{\delta_{1}}{\rho_{1}} \leq V(0)+\frac{\delta_{1}}{\rho_{1}} .
$$

Given $Q=2\left(V(0)+\delta_{1} / \rho_{1}\right)$, the closed-loop error signals $z_{1}$, $z_{2}$, and $\widetilde{W}$ will converge asymptotically to the compact sets $\Omega_{z 1}, \Omega_{z 2}$, and $\Omega_{W}$, defined by

$$
\begin{aligned}
& \Omega_{z 1}:=\left\{z_{1} \in R^{3} \mid\left\|z_{1}\right\| \leq \sqrt{Q}\right\} . \\
& \Omega_{z 2}:=\left\{z_{2} \in R^{3} \mid\left\|z_{2}\right\| \leq \sqrt{\frac{Q}{\lambda_{\min }(M)}}\right\} . \\
& \Omega_{W}:=\left\{\widetilde{W} \in R^{l \times 3} \mid\|\widetilde{W}\| \leq \sqrt{\frac{Q}{\lambda_{\text {min }}\left(\Gamma^{-1}\right)}}\right\},
\end{aligned}
$$

where $l$ denotes the neural network node number.

Therefore, $z_{1}, z_{2}$, and $\widetilde{W}_{1}, \widetilde{W}_{2}, \widetilde{W}_{3}$ are uniformly ultimately bounded. Then $\widehat{W}_{i}=\widetilde{W}_{i}+W_{i}^{*}(i=1,2,3)$ is also bounded. $z_{1}$ is the transformed error, so $e_{1}=\eta-y_{d}$ is bounded. From Assumption 2, $y_{d}$ is bounded and $\alpha$ is bounded, and thus $\eta$ and $\nu$ are bounded. The control input $\tau$ is bounded since $S(Z)$ is bounded. Thus, all signals in the closed-loop system remain bounded, and the tracking error $e_{1}=\eta-y_{d}$ not only converges to a small neighborhood of zero, but also achieves the prescribed performances.

Remark 10. To achieve the control design with no need of the initial error $e_{1}(0)$, preprocessing is needed before the operation of the controller and identifying which controller is applicable. In the paper, we have assumed that system state variable $v$ is unmeasurable. In practice, for most merchant vessels, only the heading and the position are measured. To estimate unmeasured $v$, a high-gain observer (19) is introduced. We use the Exponential function to overcome the peaking phenomenon of the high-gain observer.

\section{Simulation Studies}

Simulation studies are presented to illustrate the effectiveness of the proposed control method in this section. The following choices of the system (1) are given: $c_{13}(\nu)=-1.0948 r-$ $24.6612 v, c_{23}(v)=25.8 u, d_{11}(v)=1.3274|u|+5.8664 u^{2}+$ $0.7225, d_{22}(\nu)=8.05|r|+36.2823|u|+0.8612, d_{23}(\nu)=$ $3.450|r|+0.845|v|-0.1079, d_{32}=-0.13|r|-5.0437|v|-0.1052$, $d_{33}=0.75|r|-0.08|v|+1.9$, and $g_{1}=g_{2}=g_{3}=0$. We assume that these parameters are unknown for the control input $\tau$. The ship inertia matrix $M$ is a constant matrix and, equally with [35],

$$
M=\left[\begin{array}{ccc}
25.8000 & 0 & 0 \\
0 & 24.6612 & 1.0948 \\
0 & 1.0948 & 2.7600
\end{array}\right]
$$

$\Delta(\eta, \nu)$ is assumed to be the model uncertainties with $\Delta(\eta, v)=\left[1 ; 0.01 u^{2}+0.5 ;-0.1 r^{3}+\sin (v)\right]$.

The dead-zone nonlinearity is given as

$$
\tau=D(u)= \begin{cases}1.5(u-0.4) & u \geq 0.4 \\ 0 & -0.5<u<0.4 \\ 1.3(u+0.5) & u \leq-0.5 .\end{cases}
$$

For the state variable $x$, the performance function is chosen as

$$
\mu(t)=(0.3-0.02) \exp (-3.4 t)+0.02,
$$

where $M=1$.

For the state variables $y$, the performance function is chosen as

$$
\mu(t)=(0.25-0.01) \exp (-2.4 t)+0.01,
$$

where $M=1$.

For the state variable $\psi$, the performance function is chosen as

$$
\mu(t)=(0.25-0.01) \exp (-6.4 t)+0.01,
$$

where $M=1$.

The objective of the control is to put forward an adaptive neural control scheme to ensure that all signals in closed-loop system remain bounded, and the system output $\eta$ tracks the ideal reference trajectory $y_{d}=$ $[0.8 \sin (t), 0.8 \cos (t),-0.8 \sin (t)]^{T}$.

During the simulation, the initial conditions are $\eta(0)=$ $[0.1,0.75,-0.0873]^{T}$ and $\widehat{v}=[0,0,0]^{T} \cdot \widehat{W}_{i}(0)=0, i=1,2$, 3. $e_{1}(0)=\left[x(0)-y_{d 1}(0), y(0)-y_{d 2}(0), \psi(0)-y_{d 3}(0)\right]^{T}=$ $[0.1,-0.05,-0.0873]^{T}$. The design parameters are given as follows: $K_{1}=[12,30,24]^{T}, K_{2}=[30,40,30]^{T}, \Gamma_{1}=\operatorname{diag}\{12\}$, $\Gamma_{2}=\operatorname{diag}\{10\}$, and $\Gamma_{3}=\operatorname{diag}\{17\} . \sigma_{1}=0.02, \sigma_{2}=0.01$, and $\sigma_{3}=0.05$. The input of the RBF neural networks is $Z=$ $[\eta, \widehat{v}, \alpha, \dot{\alpha}]^{T}$ and $\widehat{W}_{i}^{T} S_{i}(Z)$ are constructed using $3^{4}$ nodes with the centers $\mu_{i}$ evenly spaced on $[-1.8,1.8] \times[-1.8,1.8] \times$ $[-1,1] \times[-1,1]$, the width being $\omega_{i}=1.1, i=1,2,3$.

Figures 2-10 show the simulation results for the prescribed performance control of the unknown ship dynamics. Figures 2, 4, and 6 show that the good tracking performances of the closed-loop system are achieved, even though no initial tracking errors are available. In Figures 3, 5, and 7 provide the curves of the tracking errors with PPC. It is obvious that the initial error $e_{1}(0)$ does not need to be known in advance. Figure 8 illustrates the time trajectory of the state variables $u, v$, and $r$ and their estimations $\widehat{u}, \widehat{v}, \widehat{r}$ by using the highgain observer. It can be seen that the peaking effect of the high-gain observer is eliminated. Figure 9 gives the norms of the adaptive laws $\widehat{W}_{1}, \widehat{W}_{2}$, and $\widehat{W}_{3}$, which demonstrates the 


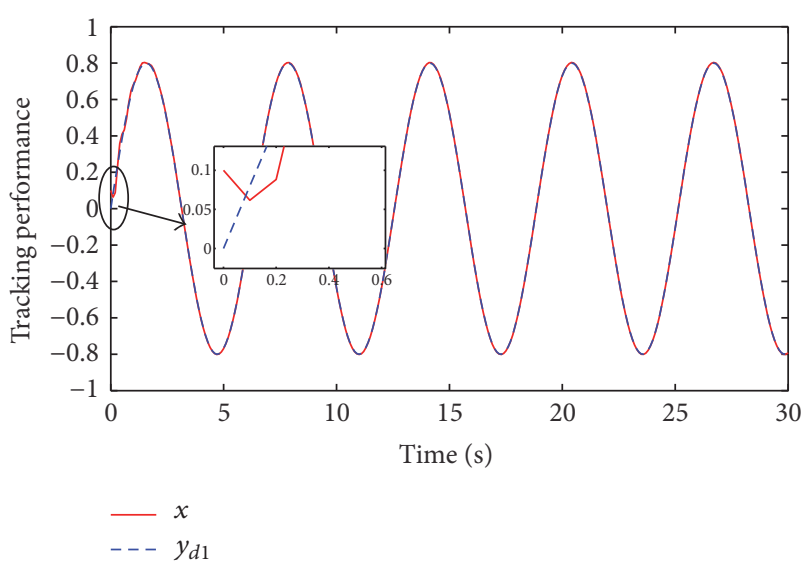

FIGURE 2: System output $x$ and reference signal $y_{d 1}$ with the positive initial error $e_{1}(0)>0$.

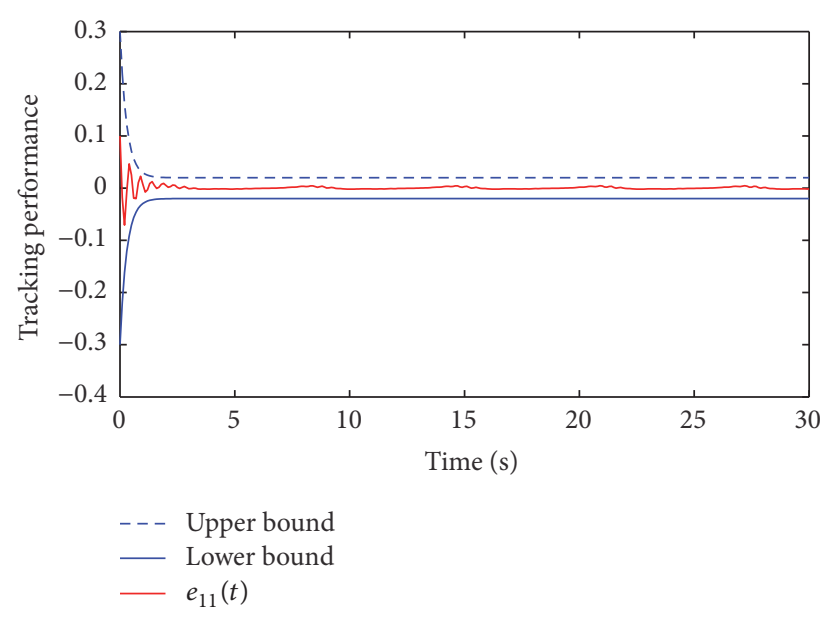

FIGURE 3: Tracking error trajectory of $x$ with the positive initial error $e_{1}(0)>0$.

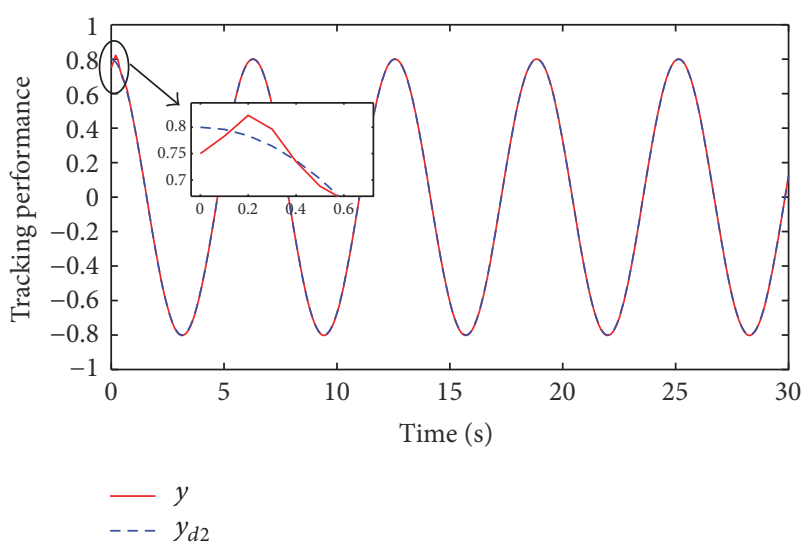

FIGURE 4: System output $y$ and reference signal $y_{d 2}$ with the negative initial error $e_{2}(0)<0$.

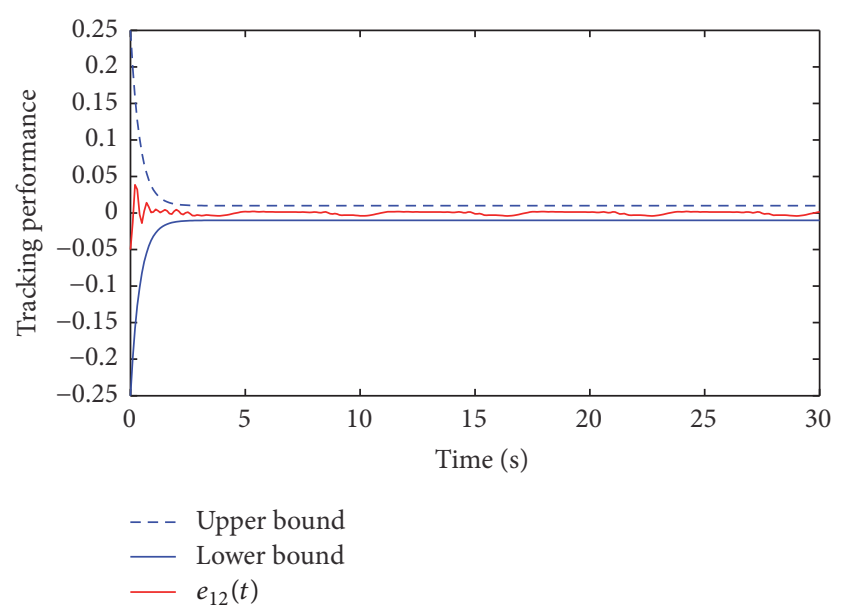

FIGURE 5: Tracking error trajectory of $y$ with the negative initial error $e_{2}(0)<0$.

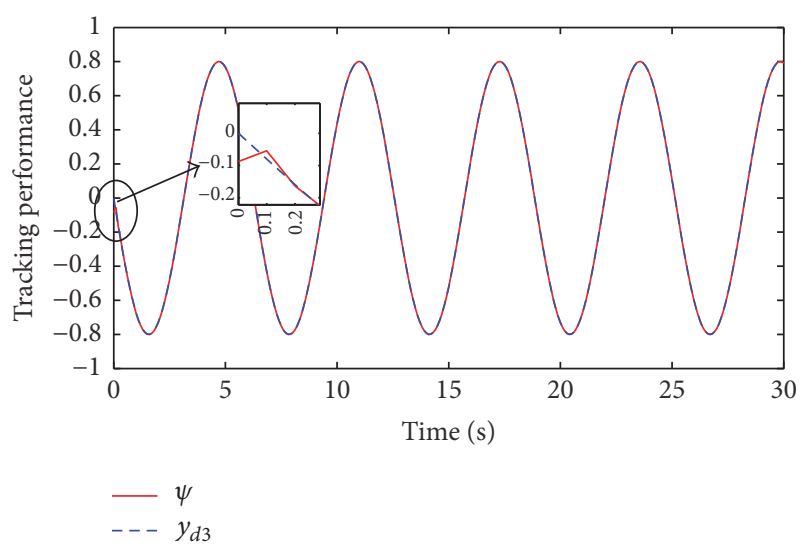

FIGURE 6: System output $\psi$ and reference signal $y_{d 3}$ with the negative initial error $e_{3}(0)<0$.

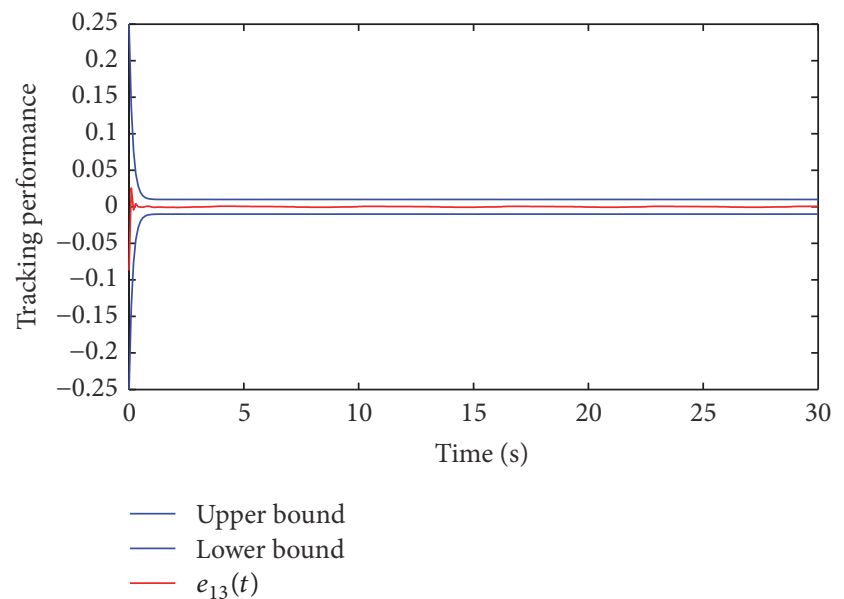

FIGURE 7: Tracking error trajectory of $\psi$ with the negative initial error $e_{3}(0)<0$. 


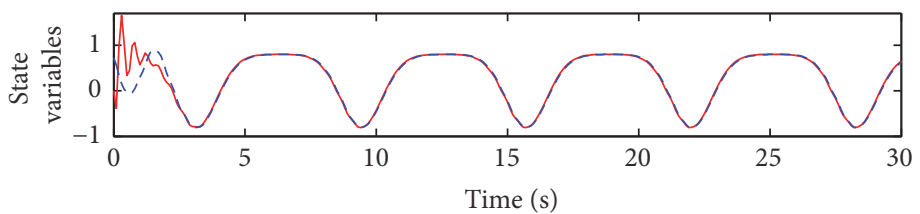

$-u$

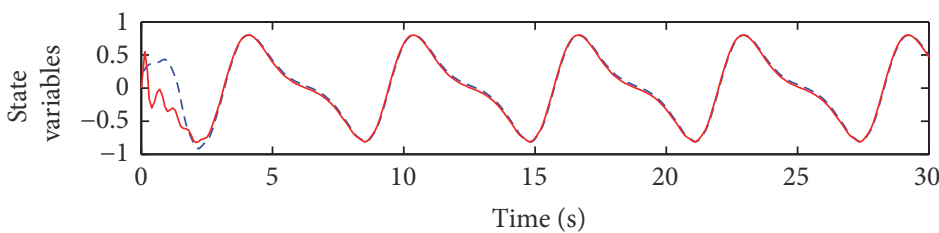

$-v$

$--\widehat{v}$

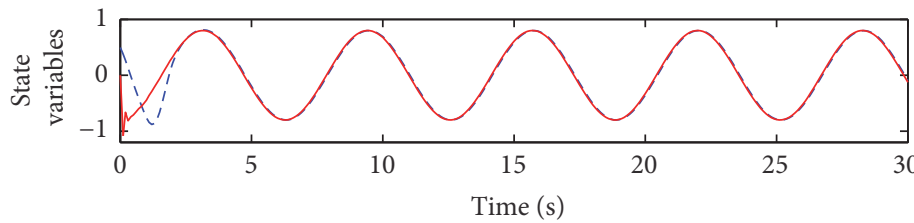

$-r$

FIgURE 8: System variables $u, v, r$ and their estimated value $\widehat{u}, \widehat{v}, \widehat{r}$.
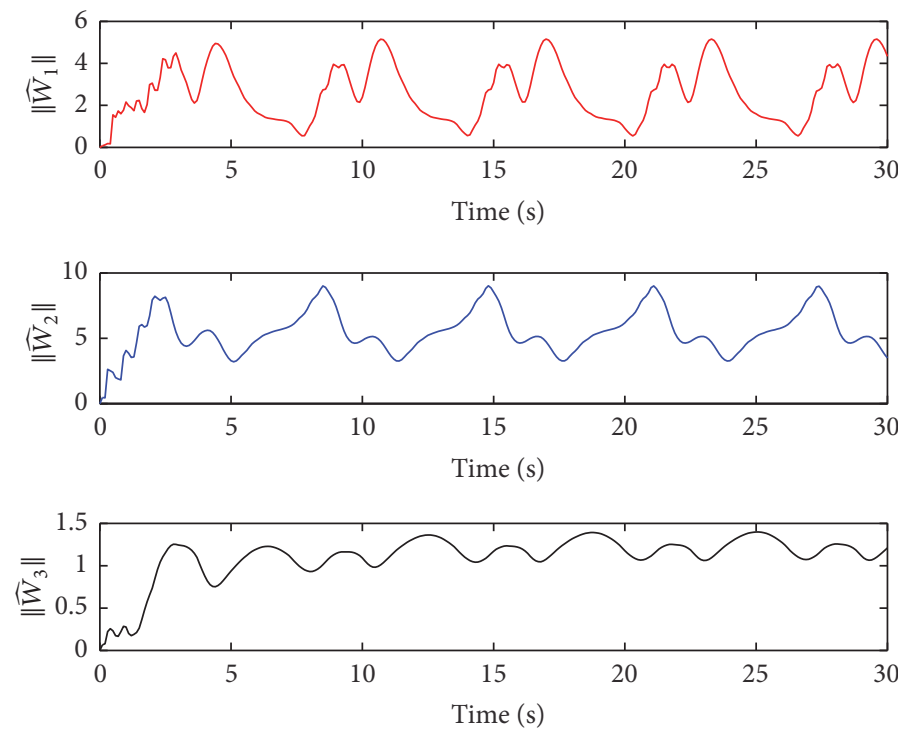

FIgURE 9: Norms of $\left\|\widehat{W}_{1}\right\|,\left\|\widehat{W}_{2}\right\|$, and $\left\|\widehat{W}_{3}\right\|$.

boundedness of the adaptive weights. The control input $\tau$ is shown in Figure 10.

\section{Conclusion}

In the paper, we investigate the problem of the tracking control with predefined performance for marine surface vessels without velocity measurements in the presence of unknown dead-zone input. RBF neural networks are used to deal with uncertain ship dynamics, a new performance function is designed with no requirement for the exact initial error, and the unmeasurable ship velocity is estimated by using a highgain observer. It has been shown that the proposed common controller can ensure that all signals in the closed-loop systems are semiglobally uniformly bounded, and tracking error converges to a predefined small neighborhood of zero. 

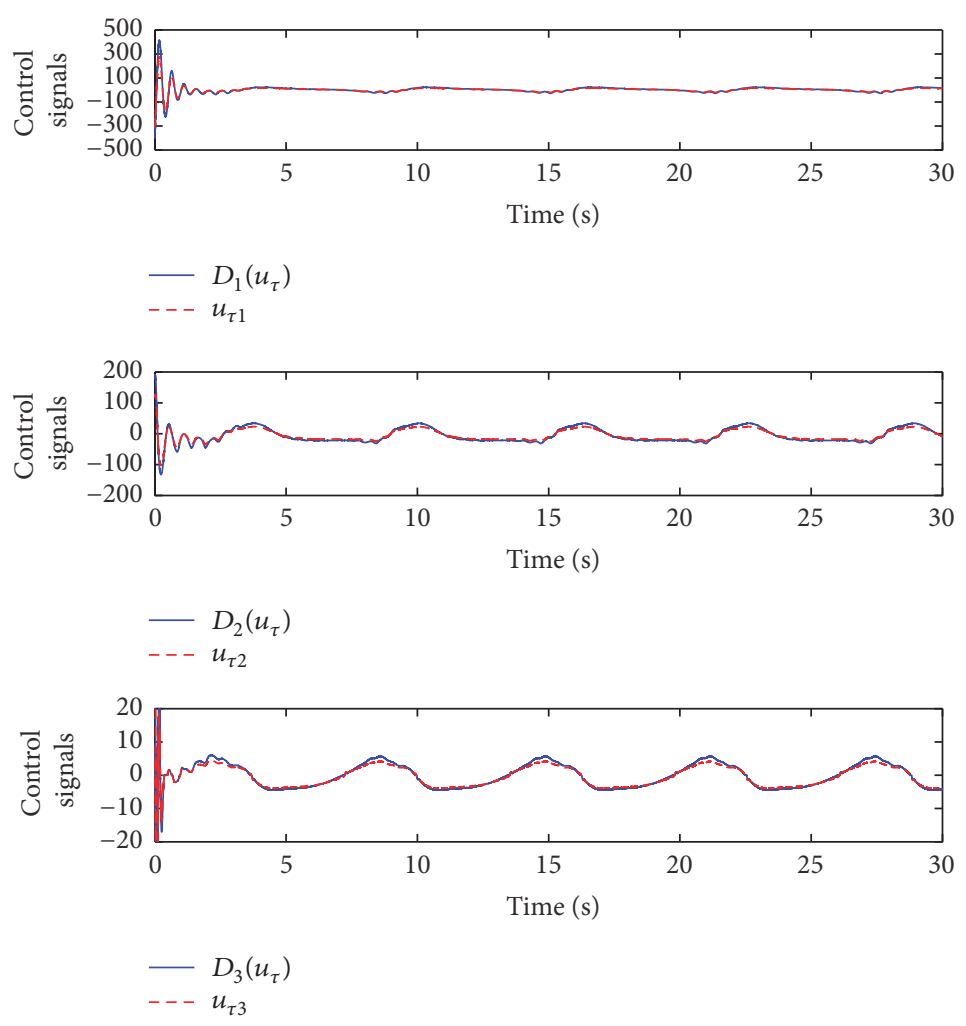

FIgURE 10: The input $u_{\tau i}$ and output $\tau_{i}(i=1,2,3)$ of the dead-zone.

\section{Competing Interests}

The authors declare that they have no competing interests.

\section{References}

[1] T. I. Fossen, Marine Control Systems: Guidance, Navigation and Control of Ships, Rigs and Underwater Vehicles, Marine Cybernetics, 2002.

[2] K. D. Doand and J. Pan, Control of Shipsand under Water Vehicles: Design for under Actuated and Nonlinear Marine Systems, Springer Science \& Business Media, 2009.

[3] R. Yu, Q. Zhu, G. Xia, and Z. Liu, "Sliding mode tracking control of an underactuated surface vessel," IET Control Theory and Applications, vol. 6, no. 3, pp. 461-466, 2012.

[4] H. Ashrafiuon, K. R. Muske, L. C. McNinch, and R. A. Soltan, "Sliding-mode tracking control of surface vessels," IEEE Transactions on Industrial Electronics, vol. 55, no. 11, pp. 40044012, 2008.

[5] S. S. Ge and C. Wang, "Adaptive neural control of uncertain MIMO nonlinear systems," IEEE Transactions on Neural Networks, vol. 15, no. 3, pp. 674-692, 2004.

[6] T.-S. Li, S.-C. Tong, and G. Feng, "A novel robust adaptivefuzzy-tracking control for a class of nonlinearmultiinput/multi-output systems," IEEE Transactions on Fuzzy Systems, vol. 18, no. 1, pp. 150-160, 2010.

[7] T.-S. Li, D. Wang, G. Feng, and S.-C. Tong, "A DSC approach to robust adaptive nn tracking control for strict-feedback nonlinear systems," IEEE Transactions on Systems, Man, and Cybernetics, Part B: Cybernetics, vol. 40, no. 3, pp. 915-927, 2010.
[8] W. He, A. O. David, Z. Yin, and C. Sun, "Neural network control of a robotic manipulator with input deadzone and output constraint," IEEE Transactions on Systems, Man, and Cybernetics: Systems, vol. 46, no. 6, pp. 759-770, 2016.

[9] Y.-J. Liu, Y. Gao, S. Tong, and Y. Li, "Fuzzy approximation-based adaptive backstepping optimal control for a class of nonlinear discrete-time systems with dead-zone," IEEE Transactions on Fuzzy Systems, vol. 24, no. 1, pp. 16-28, 2016.

[10] Y. Gao and Y.-J. Liu, "Adaptive fuzzy optimal control using direct heuristic dynamic programming for chaotic discretetime system," Journal of Vibration and Control, vol. 22, no. 2, pp. 595-603, 2016.

[11] Y.-J. Liu and S. Tong, "Adaptive fuzzy identification and control for a class of nonlinear pure-feedback mimo systems with unknown dead zones," IEEE Transactions on Fuzzy Systems, vol. 23, no. 5, pp. 1387-1398, 2015.

[12] Y.-J. Liu and S. Tong, "Adaptive fuzzy control for a class of unknown nonlinear dynamical systems," Fuzzy Sets and Systems, vol. 263, pp. 49-70, 2015.

[13] M. Chen, S. S. Ge, B. V. E. How, and Y. S. Choo, "Robust adaptive position mooring control for marine vessels," IEEE Transactions on Control Systems Technology, vol. 21, no. 2, pp. 395-409, 2013.

[14] Z. Zhao, W. He, and S. S. Ge, "Adaptive neural network control of a fully actuated marine surface vessel with multiple out putcon straints," IEEE Transactionson Control Systems Technology, vol. 22, no. 4, pp. 1536-1543, 2014.

[15] N. Wang and M. Joo Er, "Self-constructing adaptive robust fuzzy neural tracking control of surface vehicles with uncertainties and unknown disturbances," IEEE Transactions on Control Systems Technology, vol. 23, no. 3, pp. 991-1002, 2015. 
[16] K. P. Tee and S. S. Ge, "Control of fully actuated ocean surface vessels using a class of feedforward approximators," IEEE Transactions on Control Systems Technology, vol. 14, no. 4, pp. 750-756, 2006.

[17] S.-L. Dai, C. Wang, and F. Luo, "Identification and learning control of ocean surface ship using neural networks," IEEE Transactions on Industrial Informatics, vol. 8, no. 4, pp. 801-810, 2012.

[18] S.-L. Dai, M. Wang, C. Wang, and L. Li, "Learning from adaptive neural network output feedback control of uncertain ocean surface ship dynamics," International Journal of Adaptive Control and Signal Processing, vol. 28, no. 3-5, pp. 341-365, 2014.

[19] S.-L. Dai, M. Wang, and C. Wang, "Neural learning control of marine surface vessels with guaranteed transient tracking performance," IEEE Transactions on Industrial Electronics, vol. 63, no. 3, pp. 1717-1727, 2016.

[20] S. J. Yoo, J. B. Park, and Y. H. Choi, "Decentralized adaptive stabilization of interconnected nonlinear systems with unknown non-symmetric dead-zone inputs," Automatica, vol. 45, no. 2, pp. 436-443, 2009.

[21] C.-C. Hua and S. X. Ding, "Model following controller design for large-scale systems with time-delay interconnections and multiple dead-zone inputs," IEEE Transactions on Automatic Control, vol. 56, no. 4, pp. 962-968, 2011.

[22] J. Y. Cheong, S. I. Han, and J. M. Lee, "Adaptive fuzzy dynamic surface sliding mode position control for a robot manipulator with friction and deadzone," Mathematical Problems in Engineering, vol. 2013, Article ID 161325, 15 pages, 2013.

[23] G. Sun, D. Wang, and M. Wang, "Robust adaptive neural network control of a class of uncertain strict-feedback nonlinear systems with unknown dead-zone and disturbances," Neurocomputing, vol. 145, pp. 221-229, 2014.

[24] S. Tong, S. Sui, and Y. Li, "Observed-based adaptive fuzzy tracking control for switched nonlinear systems with deadzone," IEEE Transactions on Cybernetics, vol. 45, no. 12, pp. 2816-2826, 2015.

[25] Y. Li, S. Tong, and T. Li, "Observer-based adaptive fuzzy tracking control of MIMO stochastic nonlinear systems with unknown control directions and unknown dead zones," IEEE Transactions on Fuzzy Systems, vol. 23, no. 4, pp. 1228-1241, 2015.

[26] G. Cui, Z. Wang, G. Zhuang, Z. Li, and Y. Chu, "Adaptive decentralized NN control of large-scale stochastic nonlinear time-delay systems with unknown dead-zone inputs," Neurocomputing, vol. 158, pp. 194-203, 2015.

[27] C. P. Bechlioulis and G. A. Rovithakis, "Robust adaptive control of feedback linearizable MIMO nonlinear systems with prescribed performance," IEEE Transactions on Automatic Control, vol. 53, no. 9, pp. 2090-2099, 2008.

[28] C. P. Bechlioulis, Z. Doulgeri, and G. A. A. A. Rovithakis, "Neuro-adaptive force/position control with prescribed performance and guaranteed contact maintenance," IEEE Transactions on Neural Networks, vol. 21, no. 12, pp. 1857-1868, 2010.

[29] A. K. Kostarigka, Z. Doulgeri, and G. A. Rovithakis, "Prescribed performance tracking for flexible joint robots with unknown dynamics and variable elasticity," Automatica. A Journal of IFAC, the International Federation of Automatic Control, vol. 49, no. 5, pp. 1137-1147, 2013.

[30] S. I. Han and J. M. Lee, "Partial tracking error constrained fuzzy dynamic surface control for a strict feedback nonlinear dynamic system," IEEE Transactions on Fuzzy Systems, vol. 22, no. 5, pp. 1049-1061, 2014.
[31] Y. Li and S. Tong, "Prescribed performance adaptive fuzzy output-feedback dynamic surface control for nonlinear largescale systems with time delays," Information Sciences. An International Journal, vol. 292, pp. 125-142, 2015.

[32] L. Liu, Z. Wang, and H. Zhang, "Adaptive dynamic surface error constrained control for MIMO systems with backlash-like hysteresis via prediction error technique," Nonlinear Dynamics, vol. 84, no. 4, pp. 1989-2002, 2016.

[33] A. Theodorakopoulos and G. A. Rovithakis, "A simplified adaptive neural network prescribed performance controller for uncertain MIMO feedback linearizable systems," IEEE Transactions on Neural Networks and Learning Systems, vol. 26, no. 3, pp. 589-600, 2015.

[34] W. He, S. S. Ge, Y. Li, E. Chew, and Y. S. Ng, "Neural network control of a rehabilitation robot by state and output feedback," Journal of Intelligent and Robotic Systems: Theory and Applications, vol. 80, no. 1, pp. 15-31, 2014.

[35] R. Skjetne, T. I. Fossen, and P. V. Kokotović, "Adaptive maneuvering, with experiments, for a model ship in a marine control laboratory," Automatica. A Journal of IFAC, the International Federation of Automatic Control, vol. 41, no. 2, pp. 289-298, 2005.

[36] T. P. Zhang and S. S. Ge, "Adaptive dynamic surface control of nonlinear systems with unknown dead zone in pure feedback form," Automatica. A Journal of IFAC, the International Federation of Automatic Control, vol. 44, no. 7, pp. 1895-1903, 2008.

[37] R. M. Sanner and J.-J. E. Slotine, "Gaussian networks for direct adaptive control," IEEE Transactions on Neural Networks, vol. 3, no. 6, pp. 837-863, 1992.

[38] A. J. Kurdila, F. J. Narcowich, and J. D. Ward, "Persistency of excitation in identification using radial basis function approximants," SIAM Journal on Control and Optimization, vol. 33, no. 2, pp. 625-642, 1995.

[39] S. S. Ge, C. C. Hang, and T. Zhang, "Adaptive neural network control of nonlinear systems by state and output feedback," IEEE Transactions on Systems, Man, and Cybernetics, Part B: Cybernetics, vol. 29, no. 6, pp. 818-828, 1999. 


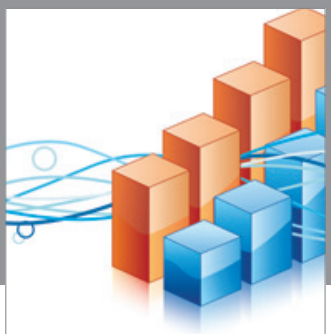

Advances in

Operations Research

vatem alat4

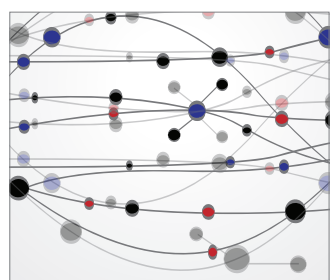

\section{The Scientific} World Journal
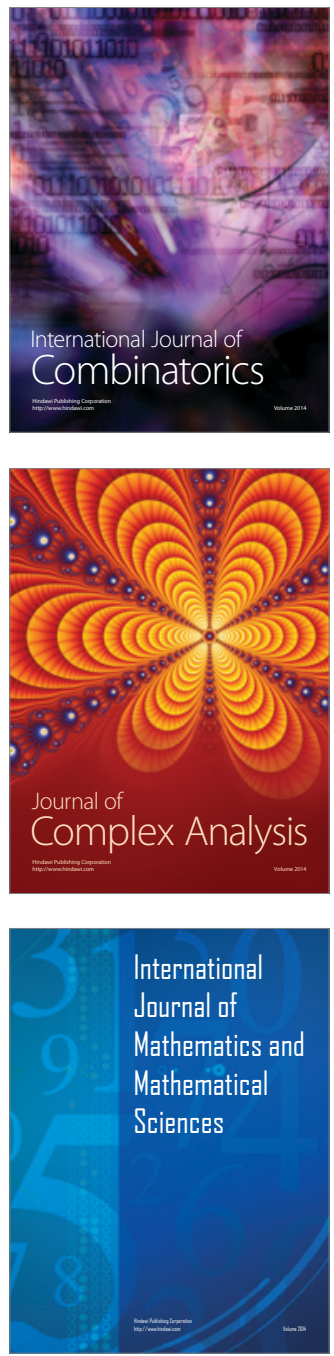
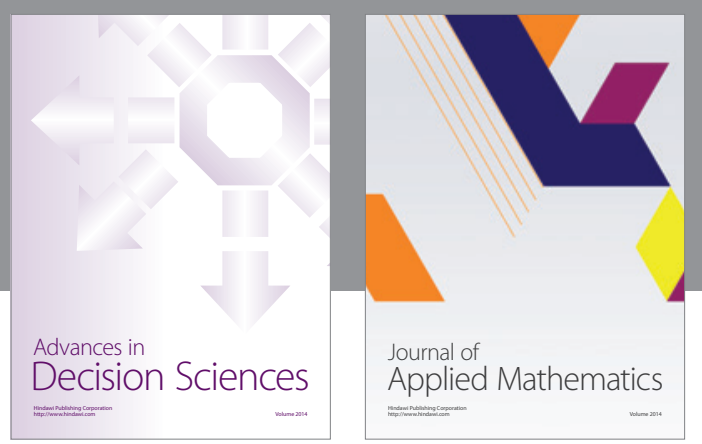

Algebra

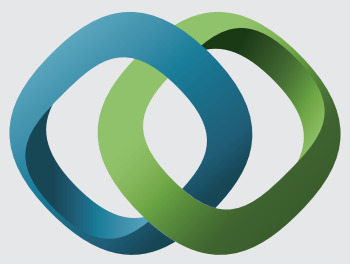

\section{Hindawi}

Submit your manuscripts at

https://www.hindawi.com
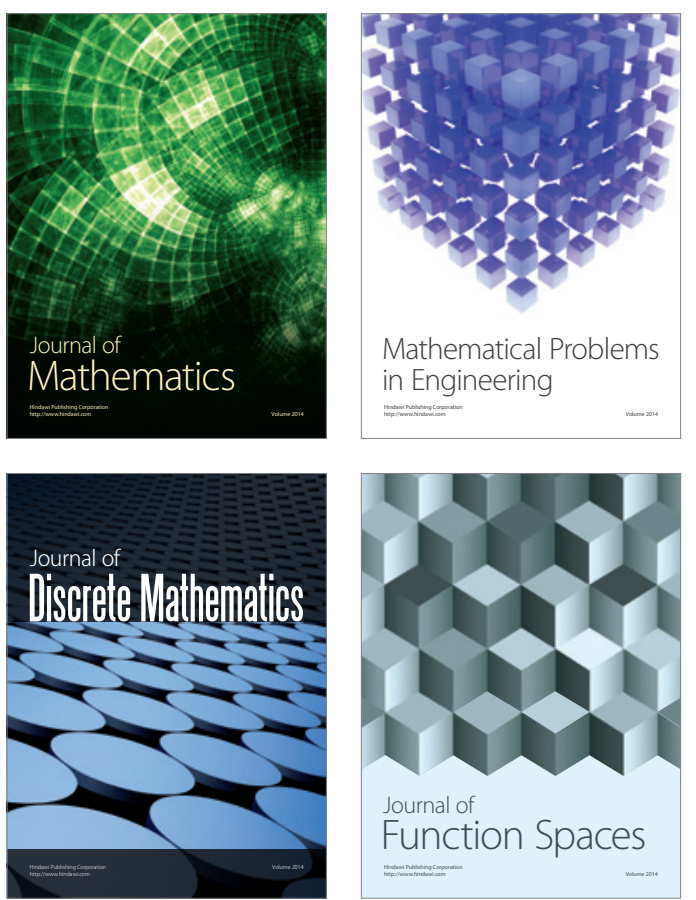

Mathematical Problems in Engineering
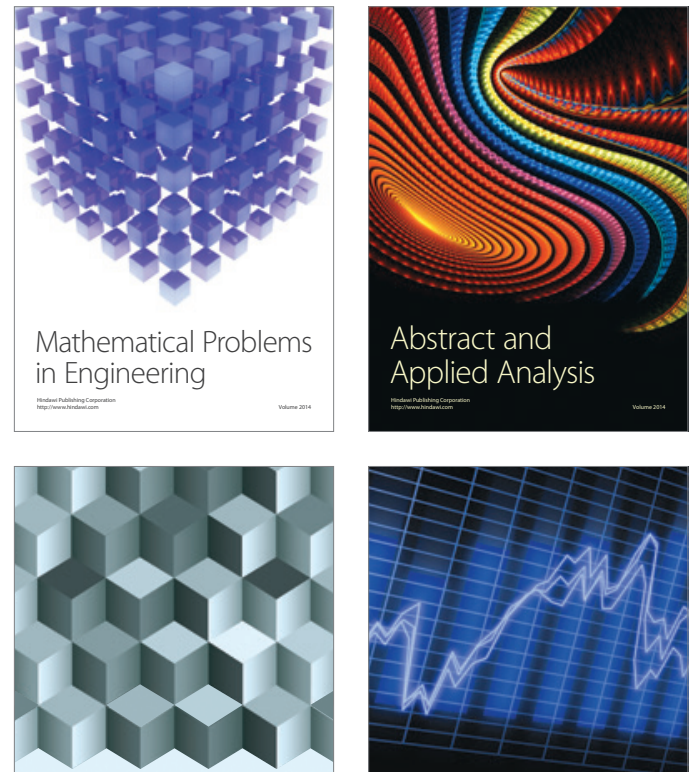

Journal of

Function Spaces

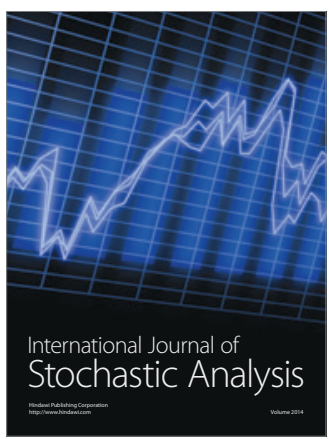

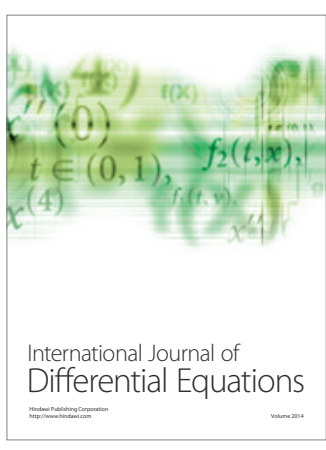
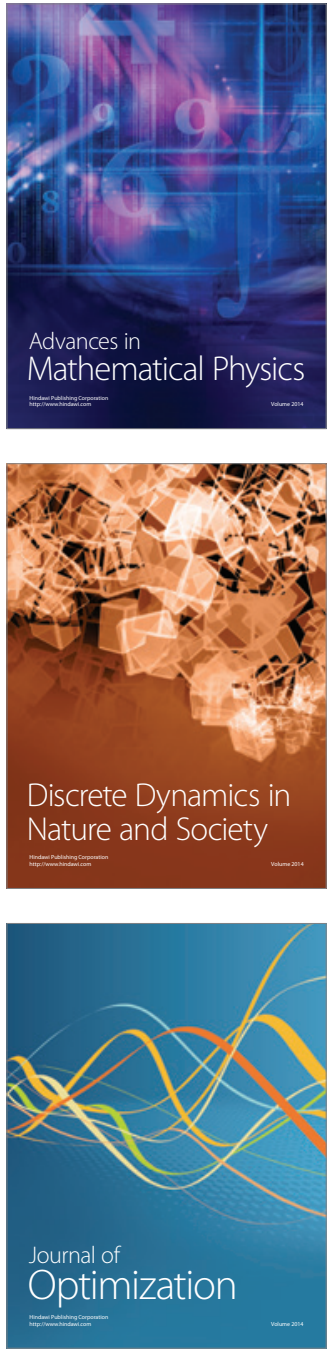